\title{
Lessons from a one-year hospital-based surveillance of acute respiratory infections in Berlin- comparing case definitions to monitor influenza
}

Matthias Nachtnebel ${ }^{1,2,3^{*}}$, Benedikt Greutelaers ${ }^{1,2,3}$, Gerhard Falkenhorst ${ }^{4}$, Pernille Jorgensen ${ }^{4}$, Manuel Dehnert ${ }^{1}$, Brunhilde Schweiger ${ }^{5}$, Christian Träder ${ }^{6}$, Silke Buda ${ }^{7}$, Tim Eckmanns ${ }^{8}$, Ole Wichmann ${ }^{4}$ and Wiebke Hellenbrand ${ }^{4}$

\begin{abstract}
Background: Surveillance of severe acute respiratory infections (SARI) in sentinel hospitals is recommended to estimate the burden of severe influenza-cases. Therefore, we monitored patients admitted with respiratory infections (RI) in 9 Berlin hospitals from 7.12.2009 to 12.12.2010 according to different case definitions (CD) and determined the proportion of cases with influenza A(H1N1)pdm09 (pH1N1). We compared the sensitivity and specificity of $\mathrm{CD}$ for capturing pandemic pH1N1 cases.
\end{abstract}

Methods: We established an Rl-surveillance restricted to adults aged $\leq 65$ years within the framework of a pH1N1 vaccine effectiveness study, which required active identification of Rl-cases. The hospital information-system was screened daily for newly admitted Rl-patients. Nasopharyngeal swabs from consenting patients were tested by PCR for influenza-virus subtypes. Four clinical CD were compared in terms of capturing pH1N1-positives among hospitalized Rl-patients by applying sensitivity and specificity analyses. The broadest case definition (CD1) was used for inclusion of Rl-cases; the narrowest case definition (CD4) was identical to the SARI case definition recommended by ECDC/WHO.

Results: Over the study period, we identified 1,025 RI-cases, of which 283 (28\%) met the ECDC/WHO SARI case definition. The percentage of SARI-cases among internal medicine admissions decreased from 3.2\% (calendar-week 50-2009) to 0.2\% (week 25-2010). Of 354 patients tested by PCR, 20 (6\%) were pH1N1-positive. Two case definitions narrower than CD1 but -in contrast to SARI- not requiring shortness of breath yielded the largest areas under the Receiver-Operator-Curve. Heterogeneity of proportions of patients admitted with Rl between hospitals was significant.

Conclusions: Comprehensive surveillance of RI cases was feasible in a network of community hospitals. In most settings, several hospitals should be included to ensure representativeness. Although misclassification resulting from failure to obtain symptoms in the hospital information-system cannot be ruled out, a high proportion of hospitalized PCR-positive pH1N1-patients (45\%) did not fulfil the SARI case-definition that included shortness of breath or difficulty breathing. Thus, to assess influenza-related disease burden in hospitals, broader, alternative case definitions should be considered.

\footnotetext{
* Correspondence: nachtnebel@post.harvard.edu

'Department of Infectious Disease Epidemiology, Robert Koch Institute, DGZ-

Ring 1, Berlin 13086, Germany

Full list of author information is available at the end of the article
} 


\section{Background}

On June 11, 2009, the World Health Organization (WHO) declared an influenza pandemic caused by influenza $\mathrm{A}(\mathrm{H} 1 \mathrm{~N} 1)$ pdm09 (referred to as pH1N1 in the following). After only low levels of pH1N1 activity during spring and summer 2009, Germany registered an increase in the number of cases starting in calendar week 42 (October) 2009. The ensuing influenza wave peaked in week 46 (November) 2009 and caused almost 250 reported deaths [1] and an estimated 2.9 (95\% CI: 2.5-3.4) million outpatient consultations [2].

Influenza is an acute viral disease of the respiratory tract. The majority of previously healthy individuals recover within 2 weeks. However, influenza can present as serious disease, for instance, as primary pneumonia, or lead to exacerbation of pre-existing cardiovascular and pulmonary disease. The very young, the elderly and patients with underlying illnesses are most at risk of developing these potentially life-threatening complications [3]. However, severe disease due to $\mathrm{pH} 1 \mathrm{~N} 1$ has also been observed in older children and young adults [4]. Estimates of $\mathrm{pH} 1 \mathrm{~N} 1$ case fatality range from $0.005 \%$ [5] in New Zealand, over 0.05\% [6] in the United States, to $1.7 \%$ in Peru [7].

WHO and the European Centre for Disease Prevention and Control (ECDC) recommend hospital-based surveillance of severe acute respiratory infections (SARI) as a tool to monitor severe disease caused by influenza [8]. This can complement surveillance of outpatient monitoring of influenza like illness (ILI) or acute respiratory illness (ARI) to cover the full spectrum of influenza-related disease. In addition, trends in the severity of a pandemic might be detected early, and risk factors for severe disease may be identified $[8,9]$. A number of countries within the WHO European region have recently established SARI surveillance of different scope and profile [10].

In Germany, systems to estimate the burden of severe disease due to influenza were not well established during the 2009 influenza pandemic. One such system, the pandemic hospital based surveillance (PIKS), was implemented in week 49 in 2009 by the Robert Koch Institute [11] to collect data on patients admitted to hospitals due to laboratory confirmed influenza, their proportion among all admitted patients, influenza cases admitted to intensive care units (ICU) as well as fatal cases. However, syndromic SARI surveillance was not routinely performed.

In the winter of 2009/10 a hospital-based pandemic influenza vaccine effectiveness study was launched. This required active case finding of all admitted patients with an acute respiratory infection to identify potential influenza cases and opened a window of opportunity to monitor the number of cases admitted with respiratory infections (RI). Our objective was to monitor the epidemiology of cases admitted with RI over time in participating hospitals as the proportion of all internal medicine and ICU admissions and to determine the proportion of cases with pH1N1. We applied different case definitions (CD), including SARI, to patients admitted with RI and tested their sensitivity and specificity for detecting $\mathrm{pH} 1 \mathrm{~N} 1$ positive patients.

\section{Methods \\ Study period}

We collected data for the period from December 7 , 2009 to December 12, 2010. We divided the study period into two segments according to the level of influenza activity detected in Berlin. Of all notified influenza cases in Berlin, 95\% were reported before week 2 in 2010, which coincided with a decline of the Germanwide consultation index of respiratory infections to background levels [12].

Elevated activity of respiratory infections was reported in week 49 (early December) 2010 and a week later, $29 \%$ of sentinel laboratory specimen sent to the Influenza Reference Centre were positive for influenza, indicating the beginning of influenza circulation in the 2010/2011 season at the end of our study period [13].

Thus, we defined study period 1 as time with significant influenza circulation which lasted from December 7, 2009 to January 10, 2010, followed by study period 2 with low or absent circulation during the spring, summer, and autumn months of 2010 (January 11, 2010 to December 12, 2010).

\section{Data collection}

The hospital information-system $\left(\mathrm{ORBIS}^{\odot}\right)$ of 9 community hospitals of the Vivantes Network for Health was screened daily for newly admitted patients. These 9 hospitals serve $\sim 30 \%$ of the Berlin population (3.4 million inhabitants) [14]. A study team comprised of 9 study nurses and additional 12 study assistants was assigned to these locations, where they accessed electronic admission entries to identify all patients admitted with acute respiratory infections to internal medicine and ICU wards. Electronic charts were checked for symptoms fulfilling the broadest case definition (CD) for RI (defined as CD1, see below) as outlined below and for date of disease onset. In cases of incomplete documentation on admission, the study team contacted nursing staff on the respective wards to obtain this information. Moreover, the study team extracted information on age, sex, place of residence, admission to ICU, and length of hospital stay. Collected data were entered into an excel spreadsheet at the hospital and transmitted to the study 
centre at the Robert Koch Institute. At the study centre, data from all 9 hospitals were merged and analyzed.

The Vivantes hospital administration contributed weekly data on the aggregated numbers of all patients admitted to the hospital network in the respective periods, with separate information on admissions to internal medicine wards and ICU.

The study population was confined to patients aged 18 to 65 years, since persons older than 65 years were not included in the vaccine effectiveness study. The study protocol was approved by the Ethics Committee of the Charité- University Medicine Berlin.

\section{Case definitions}

We applied a broad case definition (CD1) for all hospitalized RI to enable retrospective evaluation of the sensitivity and specificity of more specific (narrower) case definitions and to capture as many pH1N1-positive cases as possible (Figure 1). CD1 thus also included patients who did not fulfil some or all of the specific clinical criteria of an RI so long as an RI was documented in the chart by a physician as the suspected diagnosis. CD4 was the narrowest case definition and was

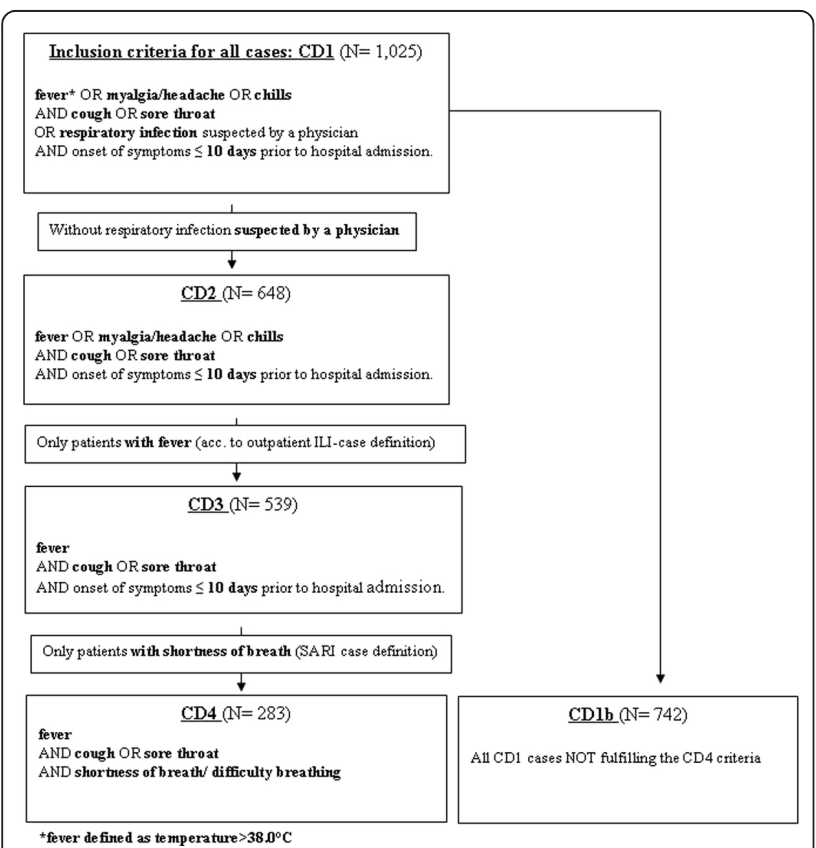

Figure 1 Flowchart of case definitions (CD) for hospitalized respiratory infections. To be included as a case in the hospitalbased surveillance of acute respiratory infections in Berlin, 2009/10, patients had to fulfil criteria of the broadest case definition (CD1). All CD1 who did not meet the CD4 (SARI) criteria were classified as CD1b. CD2 and CD3 fulfilled clinical criteria only and excluded those where clinical criteria were not fully met but a physician's

(suspected) diagnosis of RI in the chart led to inclusion. The smallest subsample of CD1 were cases fulfilling CD4 criteria (SARI as defined by $\mathrm{ECDC}$ WHO). identical to the SARI case definition as suggested by ECDC [15] and WHO [8] in 2009. In addition, we tested sensitivity and specificity of two further case definitions in terms of capturing the highest proportion of pH1N1positives among RI-patients referred to as CD2 and CD3 (Figure 1). CD2 included only those CD1 patients who fulfilled the specified clinical criteria and excluded those CD1 patients based only on a suspected diagnosis of RI. CD3 was identical to the WHO case definition for influenza like illness (ILI) [8], originally developed for the surveillance of influenza in outpatients (WHO EURO changed the ILI case definition in 2011 [16] by dropping sore throat and altering the definition of fever) (Figure 1).

Informed consent for nasopharyngeal swabbing was sought from all RI patients unless exclusion criteria as applied in the vaccine effectiveness study were present (living in a nursing home, not able to give informed consent, insufficient communication skills, contraindication for influenza vaccination). All consenting patients received a nasal and throat swab (Mastaswab; MAST Diagnostica, Reinfeld, Germany) to obtain material for real-time polymerase chain reaction (PCR) testing at the Reference Centre for Influenza. In a few patients admitted to ICU, material was collected from bronchoalveolar lavage fluids. PCR was performed as described previously, targeting the $\mathrm{M}$ gene for universal detection of influenza A viruses as well as the HA and NA genes for further subtyping including the specific detection of pH1N1 viruses [17]. This procedure was extensively validated. The limit of detection (95\% detection probability) was determined at $\sim 6$ genome equivalents per reaction indicating a high sensitivity. All PCR assays used showed $100 \%$ specificity with no cross-reactivities observed with other respiratory viruses or bacteria [17].

From April 2010 onwards, we continued to register cases from all participating hospitals, but influenza diagnosis was offered only to patients from 4 hospitals covering roughly $13 \%$ of Berlin's population, resulting in a lower proportion of swabbed patients.

\section{Statistical methods}

We calculated proportions of patients fulfilling the respective case definitions in relation to the overall number of patients admitted to internal medicine wards and ICU of the 9 hospitals. To compare medians and proportions, we used the Kruskal-Wallis and chi-square test, respectively. The Breslow-Day test was used to assess homogeneity between strata. As measures of association we calculated relative risks (RR) as well as odds ratios (OR) and their 95\% confidence intervals (CI) by univariable exact logistic regression. To evaluate trends over time we applied Poisson regression and calculated incidence rate ratios (IRR) and 95\% CI of the mean. As 
a measure of comparison for the different case definitions we utilized the area under the receiver operator curves (ROC) and 95\% CI (asymptotic normal) of the area under the curve (AUC); we compared AUC with chi-square tests. Cases testing negative were only included as such if the nasopharyngeal swab was taken within 7 days of symptom onset. A sensitivity analysis was performed using a swabbing interval of 4 days. Data analysis was performed with Stata $^{\odot}$, version 11 .

\section{Results}

Number of cases according to case definition and severity Overall, we identified 1,025 patients fulfilling the broad CD1 definition (Table 1). Of these, 283 (28\%) fulfilled CD4 criteria. The remaining 742 (72\%) cases were classified as CD1b. Case definition criteria for CD2 were met by $63 \%(\mathrm{n}=648)$ and for CD3 by $53 \%(\mathrm{n}=539)$.

We tested 354 (35\%) patients for influenza virus infection. From calendar week 50/2009 to 14/2010, 291 ARI patients were identified in the 9 participating hospitals, of whom 141 (48\%) agreed to swabbing. From calendar week 15/2010 to 49/2010, 734 ARI patients were ascertained, of whom 365 (50\%) were from the 4 hospitals in which testing continued to be offered. Of these, 196 (54\%) agreed to provide a nasopharyngeal swab. Reasons for refusal were not systematically documented, but there was no significant difference in age $(p=0.3)$ or gender distribution $(p=0.2)$ between those tested or not. In study period 1, 16 of 56 cases with test results available (29\%) tested pH1N1-positive versus 4 of 286 (1\%) in period 2 (Table 2). Overall, CD4 patients were more likely to give informed consent for nasopharyngeal swabbing than CD1b patients $(\mathrm{RR}=1.9,95 \% \mathrm{CI}$ : 1.60 2.21). The proportion of $\mathrm{pH} 1 \mathrm{~N} 1$-positives did not differ significantly between those CD4 and CD1b cases $(p=$ 0.3 ) that were tested, neither during period 1 ( $32 \%$ vs. $25 \%, p=0.5)$ nor period 2 ( $2 \%$ vs. $1 \%, p=0.7$ ).

A higher proportion of both CD1 cases and pH1N1positive patients was male (Table 1). Among CD4 cases men were older than women $(p=0.04)$, but there was no statistically significant difference between the age of men and women fulfilling the CD1 or the CD1b criteria $(p=0.7)$. The median age of CD4 patients was lower than that of CD1b patients $(p=0.05)$. Exact logistic regression, however, did not reveal a dose-response relationship between years of age and odds of fulfilling the CD4 definition $(p=0.2)$. Median age of $\mathrm{pH} 1 \mathrm{~N} 1$-positives was lower than in negatives but this was not statistically significant $(p=0.2)$.

Of the $20 \mathrm{pH} 1 \mathrm{~N} 1$ - positive cases, $55 \%$ were classified as CD4. Only four pH1N1-positive cases (20\%) were admitted to ICU, of which $3(75 \%)$ were classified as CD4. None of the 20 pH1N1-positive cases died, but pH1N1-status was positively associated with ICU-admission, although this was not statistically significant $(p=$ $0.09)$. Neither requiring mechanical ventilation $(p=0.8)$ nor length of hospital stay ( $p=0.6$, Table 1$)$ showed an association with pH1N1-status. The risk of ICU-admission $(R R=1.3,95 \% C I: 0.95-1.93)$ and of requiring mechanical ventilation $(\mathrm{RR}=1.3,95 \% \mathrm{CI}$ : $0.69-2.41)$ was similar for CD1b and CD4 cases, but median duration of hospital stay was longer for CD4 than CD1b cases $(p$ $=0.03)$. Death during hospital admission occurred in $2 \%$ of CD 4 and $4 \%$ of CD1b cases $(p=0.13)$, but data on patient outcome was missing in $23 \%$ of all included cases. The proportion of cases with missing patient outcome data did not differ by pH1N1-status, admission to ICU or CD4 versus CD1 (all p-values > 0.1).

The likelihood of hospitalized RI-patients fulfilling criteria for the narrowest case definition (CD4) was 1.3 times higher (95\%CI: $1.01-1.75)$ in study period 1 than period 2 (35\% versus $27 \%$ ). In addition, CD1-cases had a RR of 1.7 (95\%CI: 1.07-2.62) for admission to ICU and of 2.6 (95\%CI: 1.31-5.04) for requiring mechanical ventilation during period 1 compared to period 2.

Overall, 185 of 353 (52\%) influenza tests were performed more than 4 days after onset of disease and 80 of 353 (23\%) more than 7 days. During study period 1, none of the $16 \mathrm{pH} 1 \mathrm{~N} 1$-positive cases was swabbed $>7$ days after symptom onset versus 11 of 40 (28\%) pH1N1-negatives (OR $=0.13$; 95\%CI: $0-0.85 ; p=0.03)$. Four (25\%) pH1N1-positive cases, but 24 of 40 (60\%)

Table 1 Patients with respiratory infections according to case definition and pH1N1 status, demographic and clinical characteristics, hospital-based surveillance of acute respiratory infections, Berlin 2009/10

\begin{tabular}{|c|c|c|c|c|c|c|c|c|}
\hline $\begin{array}{l}\text { Cases Definition } \\
\text { (number of cases) }\end{array}$ & $\begin{array}{c}\% \\
\text { male }\end{array}$ & $\begin{array}{c}\text { Median } \\
\text { age, } \\
\text { females* }\end{array}$ & $\begin{array}{l}\text { Median } \\
\text { age, } \\
\text { males }\end{array}$ & $\begin{array}{l}\text { \% requiring } \\
\text { ICU treatment }\end{array}$ & $\begin{array}{c}\text { \% requiring } \\
\text { mechanical } \\
\text { ventilation }\end{array}$ & $\begin{array}{l}\% \text { pH1N1 positive } \\
\text { among tested }\end{array}$ & $\begin{array}{c}\text { Duration of } \\
\text { hospital stay } \\
\text { (median) }\end{array}$ & $\begin{array}{c}\text { Case } \\
\text { fatality }\end{array}$ \\
\hline CD1 $(n=1,025)$ & $61 \% *$ & $49 y$ & $51 y$ & $12 \%$ & $6 \%$ & $6 \%$ & 6 days & $4 \%$ \\
\hline CD1b $(n=742)$ & $62 \% *$ & $50 y$ & 51 y & $11 \%$ & $5 \%$ & $4 \%$ & 6 days & $4 \%$ \\
\hline CD4 $(n=283)$ & $57 \% *$ & $45 y$ & $50 y^{*}$ & $14 \%$ & $7 \%$ & $7 \%$ & 7 days & $2 \%$ \\
\hline $\begin{array}{c}\text { pH1N1-positive ( } \mathrm{n} \\
=20)\end{array}$ & $65 \%$ & $42 y$ & $46 y$ & $20 \%$ & $7 \%$ & $100 \%$ & 6 days & $0 \%$ \\
\hline
\end{tabular}

* statistically significant $(p<0.05)$ when compared to female cases

See text and Figure 1 for explanation of CD1, $1 \mathrm{~b}$ and 4 
Table 2 Absolute and relative number of patients tested positive for $\mathrm{pH} 1 \mathrm{~N} 1$ by case definition and study period, hospital-based surveillance of acute respiratory infections, Berlin, 2009/10

\begin{tabular}{llcc}
\hline & & No. tested for influenza (\%) & No. testing positive for influenza* (\%) \\
\hline CD1 & Period 1 & $60 / 110(55 \%)$ & $16(29 \%)$ \\
\cline { 2 - 4 } & Period 2 & $294 / 915(32 \%)$ & $4(1 \%)$ \\
\cline { 2 - 4 } & Total & $354 / 1,025(35 \%)$ & $20(6 \%)$ \\
\hline CD1b & Period 1 & $32 / 71(45 \%)$ & $2(1 \%)$ \\
\cline { 2 - 4 } & Period 2 & $174 / 671(26 \%)$ & $9(5 \%)$ \\
\cline { 2 - 4 } & Total & $206 / 742(28 \%)$ & $9(32 \%)$ \\
\hline CD4 & Period 1 & $28 / 39(72 \%)$ & $11(8 \%)$ \\
\cline { 2 - 4 } & Period 2 & $120 / 244(49 \%)$ & \\
\cline { 2 - 4 } & Total & $148 / 283(52 \%)$ & \\
\hline
\end{tabular}

*Patients with missing PCR results excluded

pH1N1-negative case were swabbed $>4$ days $(\mathrm{p}=0.018)$ after symptom onset.

\section{Differences between hospitals}

Absolute and relative numbers of patients admitted due to RI varied by study location. Over the entire study period, the mean proportion of CD1-cases among internal medical ward admissions was $4.9 \%$ and ranged from $3.3 \%$ to $8.4 \%$ between hospitals $(p<0.01)$. The proportion of CD4 cases among all respiratory infections was $28 \%$ overall and ranged from $19 \%$ to $44 \%$ in participating hospitals $(p<0.01)$. These results indicated heterogeneity among study locations. Neither the percentage of $\mathrm{pH} 1 \mathrm{~N} 1$ positives nor the gender distribution differed significantly by hospital among those tested $(p=0.2)$, nor did the gender distribution $(p=0.12)$. However, median age of included RI-patients ranged from 45 to 56 years by study location $(p<0.01)$.

\section{Development over time}

We calculated the weekly incidence of all CD1-cases, CD1b cases, and CD4 cases as a percentage of all admissions to internal medicine wards and of all CD1-cases admitted to ICU as a percentage of all ICU admissions (Figure 2 and 3, Table 3). All incidences showed statistically significant changes over time. Admission of CD1b cases exhibited a biphasic pattern with a decline from $5.1 \%$ in week $50 / 2009$ to a minimum of $1.4 \%$ in week 29/2010, with a weekly IRR of 0.99 (95\%CI: 0.98-1.00). Thereafter, the IRR was 1.02 (95\%CI: 1.00-1.03) per week until the end of the study period with a maximum of $5.6 \%$ in week 41 in 2010 . We observed a similar pattern for CD4 cases with an IRR of 0.96 (95\%CI: 0.940.98 ) per week to a minimum of $0.2 \%$ in week 25 in 2010; followed by a weekly IRR of 1.03 (95\%CI: 1.001.06). In contrast, the incidence of all cases among ICU admissions decreased over the entire study period with an IRR of 0.98 per week (95\%CI: 0.97-0.99).

\section{Sensitivity and specificity of case definitions}

The sensitivity and specificity of the case definitions applied to all included patients using results of PCR testing as the gold standard are shown in Table 4 both for the entire study period and separately for study period 1 with significant influenza virus circulation. The highest sensitivity was found for CD2; the highest specificity for CD4.

The area under the ROC curve was largest for CD2 in study period 1 and for CD3 during the entire study period, although not statistically significantly larger than for CD4 $(p>0.1)$ (Table 4, Figure 4). ROC areas increased only minimally if a maximal swabbing interval of 4 instead of 7 days was applied to ascertain pH1N1 negatives.

\section{Discussion}

The implementation of a surveillance system monitoring respiratory diseases at 9 Berlin hospitals initially in the

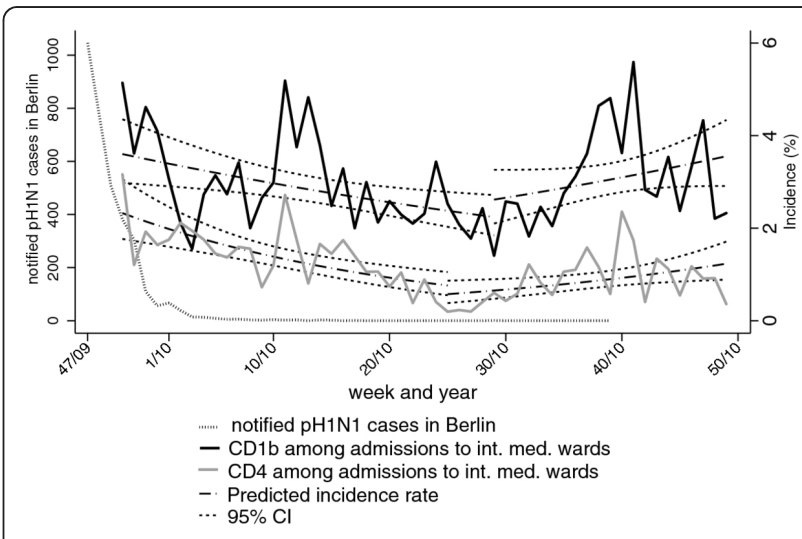

Figure 2 Incidence of hospitalized respiratory infections fulfilling criteria for CD1b and CD4 as a percentage of internal medicine ward admissions in the study hospitals per calendar week. Weekly incidences are shown together with predicted incidences and $95 \% \mathrm{Cl}$ according to Poisson regression. All pH1N1 cases notified in Berlin through the national routine surveillance system, 2009-2010 are shown for comparison. 


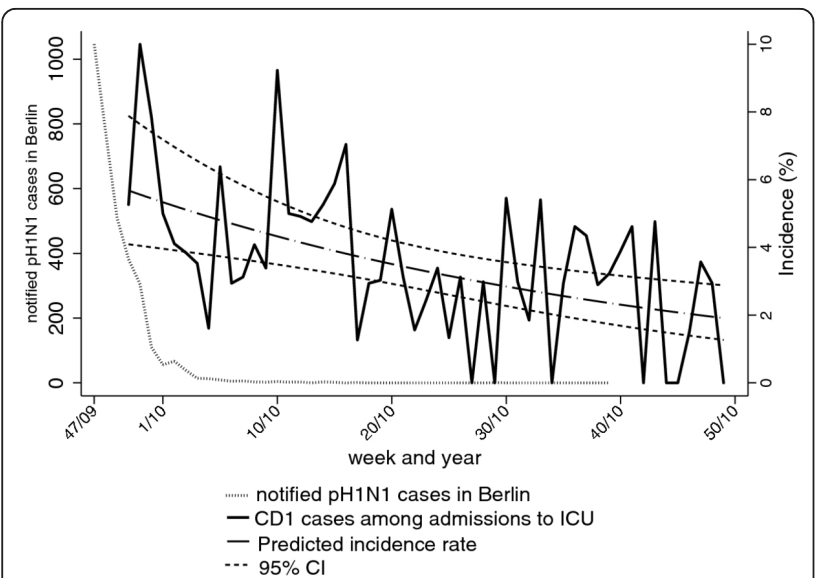

Figure $\mathbf{3}$ Incidence of respiratory infections fulfilling criteria for CD1 admitted to intensive care units (ICU) as a percentage of all admissions to ICU per calendar week. Weekly incidences are shown together with predicted incidences and $95 \% \mathrm{Cl}$ according to Poisson regression. All pH1N1 cases notified in Berlin through the national routine surveillance system, 2009-2010 are shown for comparison

context of a hospital-based vaccine effectiveness study was feasible and delivered important insights into the distribution of cases over time and according to different $\mathrm{CD}$. A large proportion of $\mathrm{pH} 1 \mathrm{~N} 1$ positives was not captured by the SARI case definition as recommended by WHO and ECDC (CD4), with alternative case definitions having a better combination of sensitivity and specificity (Table 4).

As expected, the incidence of both CD1b and CD4cases was subject to the influence of seasonality. The proportion of cases fulfilling the narrowest CD4 definition was highest during study period 1 (winter), a period with substantial influenza virus circulation, and significantly lower thereafter. This was also reflected in the higher proportion of RI patients admitted to ICU during period 1 . These findings are in keeping with a seasonal distribution of RI as described previously [18-20]. Our results suggest that the spectrum of pathogens that circulate during winter causes a higher proportion of severe cases than the pathogen mix prevailing in spring and summer. Besides influenza, many respiratory pathogens exhibit seasonal patterns with peaks during the winter, e.g. adeno- or coronavirus infections [21], but our data cannot provide insight as to which individual pathogens might cause increased morbidity. In our study almost a third of all RI-patients was positive for influenza during study period 1 , and other studies have shown that influenza viruses are among the most common pathogens leading to hospitalization [21-23] for respiratory disease or cardio-respiratory failure in the winter season [24]. Nonetheless, a significant proportion of respiratory disease is apparently caused by other pathogens; even if possible underestimation of the proportion of influenza due to late swabbing in our study is taken into account.

We found that $20 \%$ of all hospitalized pH1N1-positive patients required ICU treatment, a number within the range of previously reported figures for $\mathrm{pH} 1 \mathrm{~N} 1$ illness $[25,26]$. The higher risk of admission to ICU, mechanical ventilation and death in CD4 compared to CD1b cases was not statistically significant; this may have been due to insufficient power. Hospital stay was significantly longer in CD4 than in CD1b-cases.

The definition of CD4 (identical to the WHO/ECDC SARI case definition) captured only $55 \%$ of all hospitalized pH1N1-positive cases, and $75 \%$ of the pH1N1cases requiring ICU management. Although a higher proportion of $\mathrm{CD} 4$ than $\mathrm{CD} 1 \mathrm{~b}$-cases was pH1N1-positive, this difference was not statistically significant in either study period. Thus, we compared sensitivity and specificity of different case definitions to explore the ability of varying combinations of symptoms to capture PCR-confirmed pH1N1-influenza (gold standard) within our study population of hospitalized RI-patients. CD2, which permitted other systemic symptoms as an alternative to fever and did not require shortness of breath (SOB), had the highest sensitivity. The sensitivity of CD3, which differed from CD2 only in the strict requirement for fever, was only slightly lower. CD4 (SARI) had the lowest sensitivity. The trade-off for high sensitivity was a low specificity, which applied in reverse order to the tested case definitions. By comparing the areas under the ROC curves we took both measures -sensitivity and specificity- into account and found

Table 3 Minimum and maximum weekly incidence of respiratory infections as a proportion of internal medicine admissions and intensive care unit (ICU) admissions, respectively, according to case definition (CD), hospital-based surveillance of acute respiratory infections, Berlin, 2009/10

\begin{tabular}{lllll}
\hline Case definition & Maximum & Week & Minimum & Week \\
\hline CD1 among internal medicine admissions & $8.3 \%$ & $50 / 2009$ & $2.0 \%$ & $27 / 2010$ \\
\hline CD1b among internal medicine admissions & $5.6 \%$ & $41 / 2010$ & $1.4 \%$ & $29 / 2010$ \\
\hline CD4 among internal medicine admissions & $3.2 \%$ & $50 / 2009$ & $0.2 \%$ & $25 / 2010$ \\
\hline CD1 cases among ICU-admissions & $10.0 \%$ & $51 / 2009$ & $0 \%$ & repeatedly \\
\hline
\end{tabular}

See text and Figure 1 for explanation of CD1, $1 \mathrm{~b}$ and 4 
Table 4 Comparison of case definitions (CD) for hospitalized respiratory infections (RI) according to their ability to capture pH1N1-positive cases

\begin{tabular}{|c|c|c|c|c|c|c|c|}
\hline & \multirow[t]{2}{*}{ Case definition } & \multicolumn{3}{|c|}{ Cases from entire study period } & \multicolumn{3}{|c|}{ Cases from study period 1} \\
\hline & & Sensitivity & Specificity & $\begin{array}{l}\text { Area under ROC } \\
(95 \% \mathrm{Cl})\end{array}$ & Sensitivity & Specificity & $\begin{array}{l}\text { Area under ROC } \\
(95 \% \mathrm{Cl})\end{array}$ \\
\hline \multirow[t]{3}{*}{ Swabbing interval $\leq 4$ days } & CD4 & $55 \%$ & $59 \%$ & $\begin{array}{l}0.57 \\
(0.45-0.69)\end{array}$ & $56 \%$ & $63 \%$ & $\begin{array}{l}0.59 \\
(0.42-0.77)\end{array}$ \\
\hline & CD2 & $95 \%$ & $26 \%$ & $\begin{array}{l}0.60 \\
(0.54-0.66) \\
\end{array}$ & $100 \%$ & $44 \%$ & $\begin{array}{l}0.72 \\
(0.59-0.84) \\
\end{array}$ \\
\hline & CD3 & $90 \%$ & $41 \%$ & $\begin{array}{l}0.65 \\
(0.57-0.73) \\
\end{array}$ & $94 \%$ & $44 \%$ & $\begin{array}{l}0.69 \\
(0.55-0.83)\end{array}$ \\
\hline \multirow[t]{3}{*}{ Swabbing interval $\leq 7$ days } & CD4 & $55 \%$ & $57 \%$ & $\begin{array}{l}0.56 \\
(0.45-0.68) \\
\end{array}$ & $56 \%$ & $59 \%$ & $\begin{array}{l}0.57 \\
(0.42-0.73) \\
\end{array}$ \\
\hline & $\mathrm{CD} 2$ & $95 \%$ & $22 \%$ & $\begin{array}{l}0.59 \\
(0.53-0.64)\end{array}$ & $100 \%$ & $38 \%$ & $\begin{array}{l}0.69 \\
(0.60-0.78)\end{array}$ \\
\hline & CD3 & $90 \%$ & $35 \%$ & $\begin{array}{l}0.63 \\
(0.55-0.70)\end{array}$ & $94 \%$ & $41 \%$ & $\begin{array}{l}0.68 \\
(0.57-0.79)\end{array}$ \\
\hline
\end{tabular}

Sensitivity, specificity, area under the receiver operator curve (ROC), percentage of pH1N1-positives captured for study period 1 (significant influenza virus circulation in Berlin) and the entire study period (gold standard: PCR confirmation of pH1N1 infections)

${ }^{*}$ Cases testing negative were only included as $\mathrm{pH} 1 \mathrm{~N} 1$-negative if the interval from symptom onset to swabbing was $\leq 4$ days or $\leq 7$ days, respectively. Highest $\mathrm{ROC}$ area in bold.

comparable and most favourable results for CD2 and $\mathrm{CD} 3$, at least during the period with significant influenza circulation. However, although the area under the ROC was highest for CD4, the difference to CD2 and CD3 was not statistically significant, likely due to overall low case numbers related to the rather late start of our study towards the end of the pandemic wave. The optimal syndromic case definition for respiratory infections

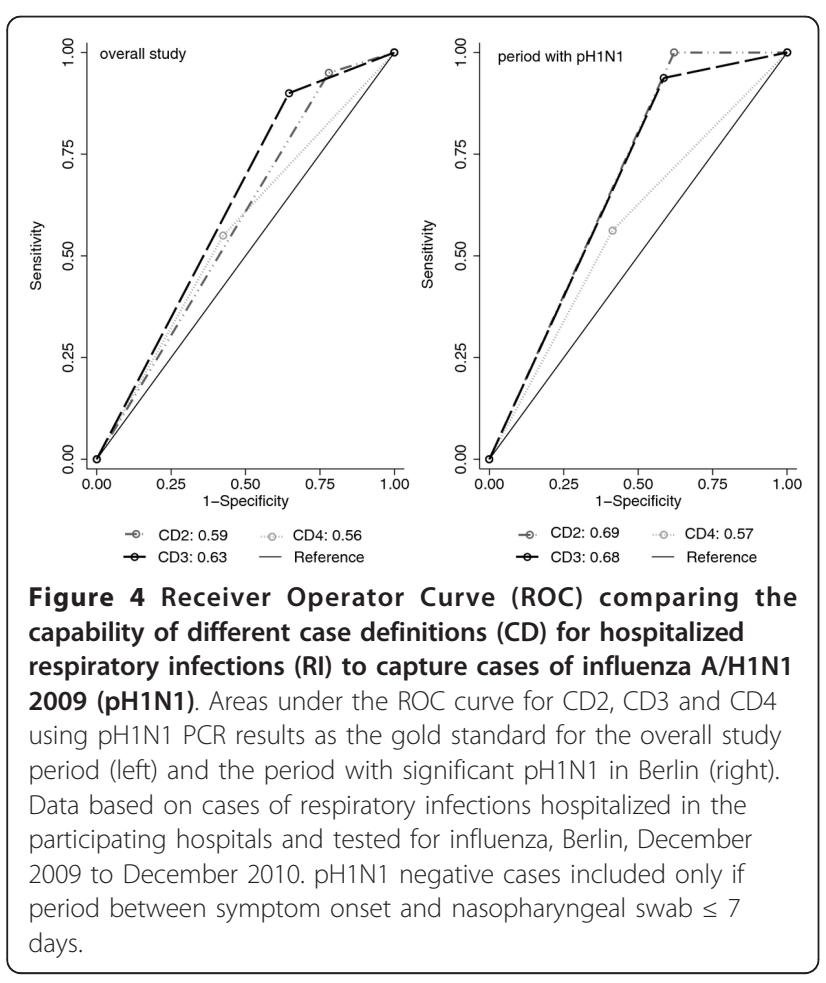

to monitor severe (hospitalized) influenza cases has been subject to repeated discussions as, for instance, the 2011 update to the ILI (CD3) case definition by WHO Europe highlights: sore throat as a symptom was dropped and a history of fever as an additional qualifying symptom added [16]. We were not able to assess the latter case definition as information on the presence of sore throat was not documented separately from cough in our study. However, our findings suggest that the broader case definitions offer advantages for surveillance of hospitalized influenza cases over the current SARI case definition as suggested by WHO/ECDC.

Hospitals showed a significant heterogeneity with respect to the proportions of patients admitted with RI. This suggests multiple study sites should be carefully selected to ensure representative surveillance of respiratory disease. Since we were interested in the trend of RI on the level of Berlin's population rather than single hospitals we did not stratify by hospital location.

Our study had some important limitations. The link to the vaccine effectiveness study led to a rather late start of our hospital-based surveillance, 2-3 weeks after the peak of the pH1N1-pandemic in Berlin and just a few weeks before it subsided. This resulted in the inclusion of a rather small number of pH1N1-positive patients. More comprehensive results would also have been possible if children and patients older than 65 years had been included in the study. Furthermore, the study ended just at the start of the 2010/2011 influenza season. Nonetheless, conduction of SARI-surveillance was found to be feasible in the framework of a hospitalbased vaccine effectiveness study and can be considered in future pandemic situations if early implementation is 
possible. At least in our setting, the presence of trained study nurses was essential for the success of SARI-surveillance due to the extra work required to extract relevant information from the hospital information-system.

Almost a quarter (23\%) of nasal and throat swabs was taken more than 7 days after symptom onset. This proportion differed by pH1N1-status: $5 \%$ among pH1N1positives versus $24 \%$ among negatives $(p=0.052)$, suggesting potential misclassification of $\mathrm{pH} 1 \mathrm{~N} 1$-positives as negative. However, other studies reported shedding of influenza virus for 6 to 9 days and, moreover, that shedding in hospitalized patients and with underlying illness appears to be longer than in households [27-32]. In addition, we cannot rule out misclassification from inability to obtain or document symptoms in the hospital information-system. This could also be an explanation for the lower sensitivity of the CD4 case definition. Clinical information on underlying disease, which might serve to explain gender differences in regards of admission to ICU, was only available for patients included in the vaccine effectiveness study (results not shown), but was not available for all patients in the SARI-surveillance. Finally, our study was confined to one large city and is therefore not necessarily representative for other populations.

\section{Conclusions}

Overall, experience with SARI-surveillance is scarce and urgently needed to establish the best practise of monitoring disease burden due to severe influenza. Although our surveillance began after the peak of the pandemic, our results permitted the depiction of decreasing influenza activity at the end of the pandemic wave. Our study added valuable insights, such as into the overall burden of RI patients requiring hospitalization, ICU admission and mechanical ventilation in periods with and without influenza virus circulation. In addition, our ROC comparison suggested that slightly broader case definitions than the WHO/ECDC SARI definition can capture a higher proportion of $\mathrm{pH} 1 \mathrm{~N} 1$ positive cases and might therefore be more appropriate for surveillance of hospitalized influenza during epidemic waves or a pandemic.

\footnotetext{
Acknowledgements

We thank the following colleagues for providing valuable assistance: Kristina Allers, Angelika Dittrich, Nadine Fiedler, Kaija Götte-Groschewski, Katrin Helge, Annemarie Mahlzahn, Christiane Rabatzek, Feyza Turfan, Regina Wosnitza for recruiting cases and hospital controls. Nora Döhnert, Fritz Feistner, Sebastian Flegel, Diana Hoffmann, Susanne Schink, Svea Schwerdfeger, Sverre Schwerdfeger, Malik Seddek, Arman Tavakoli-Kolagari, Anna-Lisa Wellhäuser, Phillip Weise for case and control recruitment or data entry. Susanne Pinkwart, Barbara Biere, Mareen Adam for technical assistance in the Influenza Reference Centre. Babette Kleinstück and Sebastian Heck, who acted as data managers. Matthias an der Heiden for statistical advice. Dorothea Matysiak-Klose and Melanie Schuster for assistance in coordinating
}

case and control recruitment in the initial stages of the study. Professor Alfred Holzgreve, director of the Vivantes Clinics in Berlin and the staff of all participating hospitals of Vivantes for their support. Financial support was provided by the German Ministry of Health.

\section{Author details}

'Department of Infectious Disease Epidemiology, Robert Koch Institute, DGZRing 1, Berlin 13086, Germany. ${ }^{2}$ Post Graduate Training in Applied Epidemiology, Robert Koch Institute, Berlin, Germany. ${ }^{3}$ European Programme for Intervention Epidemiology Training (EPIET), European Centre for Disease Prevention and Control (ECDC), Stockholm, Sweden. ${ }^{4}$ Immunization Unit, Robert Koch Institute, Berlin, Germany. ${ }^{5}$ National Reference Centre for Influenza, Robert Koch Institute, Berlin, Germany. 'Vivantes Clinic, Berlin, Germany. ${ }^{7}$ Respiratory Disease Unit, Robert Koch Institute, Berlin, Germany. ${ }^{8}$ Surveillance Unit, Robert Koch Institute, Berlin, Germany.

\section{Authors' contributions}

MN drafted the manuscript, was involved in the data collection process, and was responsible for the data analysis and interpretation. BG, GF, PJ, CT, OW, and $\mathrm{WH}$ were involved in the study design, data collection, and review of the draft manuscript. MD, TE, and SB had made significant contributions to the analytical part, interpretation of results, and review of the manuscript. BS was responsible for the laboratory testing of specimen, interpretation of laboratory results, and reviewed the draft manuscript. All authors read and approved the final manuscript.

\section{Competing interests}

The authors declare that they have no competing interests.

Received: 12 September 2011 Accepted: 27 March 2012

Published: 27 March 2012

\section{References}

1. Buda S, Kopke K, Haas W: Epidemiological characteristics of the influenza pandemic (H1N1) 2009 in Germany based on the mandatory notification of cases. Bundesgesundheitsblatt Gesundheitsforschung Gesundheitsschutz 2010, 53(12):1223-1230.

2. Arbeitsgemeinschaft Influenza (AGl): Bericht zur Epidemiologie der Influenza in Deutschland Saison 2009/10 2010.

3. Rothberg MB, Haessler SD, Brown RB: Complications of viral influenza. Am J Med 2008, 121(4):258-264.

4. Rothberg MB, Haessler SD: Complications of seasonal and pandemic influenza. Crit Care Med 2010, 38(4 Suppl):e91-97.

5. Baker MG, Wilson N, Huang QS, Paine S, Lopez L, Bandaranayake D, Tobias M, Mason K, Mackereth GF, Jacobs M, Thornley C, Roberts S, McArthur C: Pandemic influenza A(H1N1)v in New Zealand: the experience from April to August 2009. Euro Surveill 2009, 14(34), pii = 19319 http://www.eurosurveillance.org/ViewArticle.aspx?Articleld=19319.

6. Presanis AM, Lipsitch M, Daniela De A, Swine Flu Investigation Team NYCDoH, Mental H, Hagy A, Reed C, Riley S, Cooper B: The severity of pandemic H1N1 influenza in the United States, April - July 2009. PLoS Curr 2009, 1:RRN1042.

7. Gomez J, Munayco C, Arrasco J, Suarez L, Laguna-Torres V, Aguilar P, Chowell G, Kochel T: Pandemic influenza in a southern hemisphere setting: the experience in Peru from May to September, 2009. Euro Surveill 2009, 14(42), pii = 19371 http://www.eurosurveillance.org/ ViewArticle.aspx?Articleld=19371.

8. World Health Organisation, Regional Office for Europe: Denmark: WHO European guidance for influenza surveillance in humans. 2009.

9. European Centre for Disease Prevention and Control: Weekly influenza surveillance overwiew. ECDC. Stockholm; 2009 [http://ecdc.europa.eu/en/ healthtopics/seasonal_influenza/epidemiological_data/pages/ weekly_influenza_surveillance_overview.aspx].

10. World Health Organisation: Overview of sentinel systems for hospitalized severe acute respiratoryinfections (SARI) presented in the weekly EuroFlu surveillance bulletin.Edited by: WHO 2011, 320[http://www.euroflu. org], Accessed 15 June 2011.

11. Pandemische Influenza A/H1N1 Krankenhaus Surveillance (PIKS): Erste Ergebnisse:. [http://www.rki.de/DE/Content/Infekt/EpidBull/epid_bull_form. html].

12. Robert Koch Institute: RKI-Ratgeber Influenza. 2009. 
13. Arbeitsgemeinschaft Influenza (AGI): Influenza Wochenbericht. Institut RK. Berlin; 2010 [http://influenza.rki.de/].

14. Fläche und Bevölkerung. [http://www.statistik-portal.de/Statistik-Portal/ de_jb01_jahrtab1.asp].

15. European Centre for Disease Prevention and Control: Overview of surveillance of influenza 2009/2010 in the EU/EEA. 2009 [http://ecdc. europa.eu/en/publications/Publications/ 0909_TED_Overview_of_Surveillance_of_Influenza_2009-2010_in_EU-EEA. pdf].

16. World Health Organisation, Regional Office for Europe: Denmark: WHO Regional Office for Europe guidance for sentinel influenza surveillance in humans. 2011 [http://www.euro.who.int/_data/assets/pdf_file/0020/ 90443/E92738.pdf].

17. Schulze M, Nitsche A, Schweiger B, Biere B: Diagnostic approach for the differentiation of the pandemic influenza $A(\mathrm{H} 1 \mathrm{~N} 1) \mathrm{v}$ virus from recent human influenza viruses by real-time PCR. PLoS One 2010, 5(4):e9966.

18. Dodek PM, Norena M, Keenan SP, Teja A, Wong H: Intensive care unit admissions for community-acquired pneumonia are seasonal but are not associated with weather or reports of influenza-like illness in the community. J Crit Care 2010.

19. Elliot AJ, Cross KW, Fleming DM: Acute respiratory infections and winter pressures on hospital admissions in England and Wales 1990-2005. $J$ Public Health (Oxf) 2008, 30(1):91-98.

20. Marrie TJ, Huang JQ: Epidemiology of community-acquired pneumonia in Edmonton, Alberta: an emergency department-based study. Can Respir J 2005, 12(3):139-142.

21. Jennings LC, Anderson TP, Beynon KA, Chua A, Laing RT, Werno AM, Young SA, Chambers ST, Murdoch DR: Incidence and characteristics of viral community-acquired pneumonia in adults. Thorax 2008, 63(1):42-48.

22. Minosse C, Selleri M, Zaniratti MS, Cappiello G, Longo R, Schifano E, Spano A, Petrosillo N, Lauria FN, Puro V, et al: Frequency of detection of respiratory viruses in the lower respiratory tract of hospitalized adults. $J$ Clin Virol 2008, 42(2):215-220.

23. Schanzer DL, Langley JM, Tam TW: Role of influenza and other respiratory viruses in admissions of adults to Canadian hospitals. Influenza Other Respi Viruses 2008, 2(1):1-8.

24. Carrat F, Leruez-Ville M, Tonnellier M, Baudel JL, Deshayes J, Meyer P, Maury E, Galimand J, Rouzioux C, Offenstadt G: A virologic survey of patients admitted to a critical care unit for acute cardiorespiratory failure. Intensive Care Med 2006, 32(1):156-159.

25. Poeppl W, Hell M, Herkner H, Stoiser B, Fritsche G, Schurz-Bamieh N, Poeppl G, Gattringer R, Jones N, Maass M, et al: Clinical aspects of 2009 pandemic influenza A (H1N1) virus infection in Austria. Infection 2011, 39(4):341-52.

26. Skarbinski J, Jain S, Bramley A, Lee EJ, Huang J, Kirschke D, Stone A Wedlake T, Richards SM, Page S, et al: Hospitalized patients with 2009 pandemic influenza A ( $\mathrm{H} 1 \mathrm{~N} 1)$ virus infection in the United StatesSeptember-October 2009. Clin Infect Dis 2011, 52(Suppl 1):S50-59.

27. Leekha S, Zitterkopf NL, Espy MJ, Smith TF, Thompson RL, Sampathkumar P: Duration of influenza A virus shedding in hospitalized patients and implications for infection control. Infect Control Hosp Epidemiol 2007, 28(9):1071-1076

28. Suess T, Buchholz U, Dupke $S$, Grunow R, an der Heiden M, Heider A, Biere B, Schweiger B, Haas W, Krause G: Shedding and transmission of novel influenza virus A/H1N1 infection in households-Germany, 2009. Am J Epidemiol 2010, 171(11):1157-1164.

29. Bhattarai A, Villanueva J, Palekar RS, Fagan R, Sessions W, Winter J, Berman L, Lute J, Leap R, Marchbanks T, et al: Viral shedding duration of pandemic influenza A H1N1 virus during an elementary school outbreak-Pennsylvania, May-June 2009. Clin Infect Dis 2011, 52(Suppl 1): S102-108.

30. Kay M, Zerr DM, Englund JA, Cadwell BL, Kuypers J, Swenson P, KwanGett TS, Bell SL, Duchin JS: Shedding of Pandemic (H1N1) 2009 Virus among Health Care Personnel, Seattle, Washington, USA. Emerg Infect Dis 2011, 17(4):639-644.

31. Na S, Chong YP, Kim MN, Kim WY, Kim W, Hong SB, Lim CM, Koh Y, Kwon JW, Hong SJ, et al: Duration of viral shedding in patients admitted to hospital with pandemic influenza A/H1N1 2009 infection. J Med Virol 2011, 83(1):5-9.

32. Lee N, Chan PK, Hui DS, Rainer TH, Wong E, Choi KW, Lui GC, Wong BC, Wong RY, Lam WY, et al: Viral loads and duration of viral shedding in adult patients hospitalized with influenza. J Infect Dis 2009, 200(4):492-500.

\section{Pre-publication history}

The pre-publication history for this paper can be accessed here: http://www.biomedcentral.com/1471-2458/12/245/prepub

doi:10.1186/1471-2458-12-245

Cite this article as: Nachtnebel et al:: Lessons from a one-year hospitalbased surveillance of acute respiratory infections in Berlin- comparing case definitions to monitor influenza. BMC Public Health 2012 12:245.

\section{Submit your next manuscript to BioMed Central and take full advantage of:}

- Convenient online submission

- Thorough peer review

- No space constraints or color figure charges

- Immediate publication on acceptance

- Inclusion in PubMed, CAS, Scopus and Google Scholar

- Research which is freely available for redistribution 